\title{
Determinantes de los procesos de acreditación de las facultades y escuelas de medicina de México
}

\author{
Determinants of the accreditation processes of the medical \\ facultiesand schools of Mexico \\ Determinantes dos processos de acreditação das faculdades \\ demedicinae escolas do México
}

\section{ELVIA ENGRACIA PATRICIA HERRERA GUTIÉRREZ1}

\begin{abstract}
RESUMEN
El objetivo principal de esta investigación es explicar la importancia de la calidad de la Educación Médica en los procesos de acreditación en México. Para ello, se conceptúa en la lógica formal que permite explicar los determinantes de los procesos de acreditación de las Escuelas y Facultades de Medicina en México. Mide y cuantifica la eficacia de los procesos de acreditación para el logro de los objetivos formativos. Asimismo, investiga la relación sujeto-objeto basado en el paradigma positivista, ya que busca la relación causa-efecto que permite explicar el porqué de los hechos y no el para qué. La conveniencia de este estudio radica en que aporta elementos de análisis concluyentes y aplicables para mejorar la calidad de la educación médica en México. También, que egresen profesionales mejor preparados; con ética, gran sentido humano, vocación por la investigación y con motivación para actualizar sus capacidades medicas acorde a la medicina moderna que se ejerce en México y el resto del mundo.
\end{abstract}

Palabras clave: Acreditación; evaluación; calidad; ética; investigación.

\begin{abstract}
The main objective of this research is to explain the importance of the quality of Medical Education in the accreditation processes in Mexico. For this, it is conceptualized in the formal logic that allows to explain the determinants of the accreditation processes of the Schools and Faculties of Medicine in Mexico. It measures and quantifies the effectiveness of the accreditation processes for achieving the training objectives. Likewise, it investigates the subject-object relationship based on the positivist paradigm, since it seeks the cause-effect relationship that allows explaining the why of the facts and not the why. The convenience of this study lies in the fact that it provides conclusive and applicable elements of analysis to improve the quality of medical education in Mexico. Also, that better prepared
\end{abstract}


professionals graduate; with ethics, great human sense, vocation for research and with motivation to update their medical capabilities according to modern medicine practiced in Mexico and the rest of the world.

Keywords: Accreditation; evaluation; quality; ethics; research.

\section{RESUMO}

O principal objetivo desta pesquisa é explicar a importância da qualidade da educação médica nos processos de acreditação no México. Para isso, conceitua-se na lógica formal que permite explicar os determinantes dos processos de credenciamento das Escolas e Faculdades de Medicina do México. Ele mede e quantifica a eficácia dos processos de acreditação para atingir os objetivos de treinamento. Da mesma forma, investiga a relação sujeito-objeto com base no paradigma positivista, pois busca a relação causa-efeito que permite explicar o porquê dos fatos e não o porquê. A conveniência deste estudo reside no fato de fornecer elementos de análise conclusivos e aplicáveis para melhorar a qualidade da educação médica no México. Além disso, profissionais mais bem preparados se formam; com ética, grande senso humano, vocação para a pesquisa e com motivação para atualizar suas capacidades médicas de acordo com a medicina moderna praticada no México e no resto do mundo.

Palavras-chave: Acreditação; avaliação; qualidade; ética; pesquisa.

\section{INTRODUCCIÓN}

El Consejo Mexicano para la Acreditación de la Educación Médica (Comaem) se fundó en enero de 2002, bajo convenio elaborado por la Asociación Mexicana de Facultades y Escuelas de Medicina (Amfem) presidida por la doctora Patricia Herrera Gutiérrez y el Consejo para la Acreditación de la Educación Superior A.C. (Copaes) siendo presidente el doctor Hugo Aréchiga Urtuzuastegui. Dicho acuerdo se llevó a cabo en virtud de que la acreditación de las Escuelas y Facultades de Medicina inició en la Asociación Mexicana de Facultades y Escuelas de Medicina (Amfem) en el año de 1993 bajo el Programa de Calidad de la Educación Médica. La primera acreditación se otorgó a la Facultad de Medicina de la Universidad Autónoma del Estado de México, cuya verificación se desarrolló en 1995; concluyendo el dictamen en 1996. Los objetivos del Programa de Calidad de Amfem se cumplieron con acierto y decisión durante seis años.

La acreditación es el reconocimiento público que otorga el Comaem siempre que el programa de medicina que se ofrece a la sociedad, cumple con determinados criterios y parametros de calidad, reconociéndolos pública y formalmente, y cuyo objetivo sea fomentar la mejora contínua a través de las observaciones o recomendaciones que se formulan, ayudando a las instituciones educativas de medicina a elaborar sus estratégias con el fin de elevar la calidad de sus procesos e iniciar las reformas conducentes para hacer lo acorde a las necesidades sociales y coadyuvar en estrategias y herramientas de planeación que hagan más efectiva y eficiente la gestión de las Instituciones de Educación Superior en aras de que cumplan cabalmente con su compromiso social y puedan afrontar exitosamente los retos de la globalización y la internacionalización de la práctica profesional. 
Básicamente, se presenta el plan de estudios aprobado por los cuerpos colegiados, respaldado por su legislación institucional y congruente con la misión de la institución, se demuestra que se lleva a cabo un buen sistema en la selección y admisión de alumnos a cargo de un comité bajo reglamento, e igualmente que la contratación de la planta docente esté amparada por el marco legal de la institución y cumpla las necesidades académicas operativas acorde al plan de estudios, los criterios de evaluación deben ser congruentes y oportunamente conocidos por los implicados; posteriormente el resultado será tomado en cuenta para retroalimentar los objetivos de la Institución.

Las escuelas y facultades de Medicina se evalúan para lograr su acreditación en lo referente a: orientación institucional y gobierno, tomando en cuenta la pertenencia a universidades o Institutos de Educación Superior y comprueba que es un programa educativo legalmente reconocido con fines académicos que garantizan un ambiente académico propicio para la educación, investigación y desarrollo integral del futuro médico.

La presente investigación plantea abrir varias líneas de investigación relacionadas al proceso de acreditación (evaluación de los estándares de calidad).

El proceso de organización y administración en la esfera educativa describe específicamente cada uno de los puntos que pueden ser en la presente investigación variables que les den fortaleza a los resultados:

- La organización institucional. Mercadotecnia institucional.

- Formación, superación y desarrollo del profesorado. Innovación educativa.

- Planeación estratégica educacional. Vínculo entre docencia, investigación y sector productivo y de los servicios.

- Procesos de centralización-descentralización.

- Evaluación institucional (interna y externa). Técnicas de planeación en educación.

- Supervisión educativa. Legislación Educativa de México.

- Bases jurídicas de la educación. Relación humana en educación.

La transformación histórico-político-social, la pueden generar las dos corrientes de la investigación más utilizadas en educación en el siglo XX, la Aristotélica y Galileana, la importancia radica en que conociéndolas podemos saber bajo que enfoques particulares cada uno interpreta la realidad.

En la observación y experimentación minuciosa sobre los fenómenos y/u objetos de estudio la tradición Galileana, se remonta a Pitágoras y Platón y la consolida Galileo Galilei (15641642). Se tomó a la ciencia como explicación causal y esto constituye la base de la llamada ciencia moderna, la cual se va fortaleciendo desde el siglo XIII al XVI. En 1638 Galileo cristalizó el nuevo método científico y, a partir de ese momento, el mundo es visto como una sucesión de fenómenos que obedecen a leyes (modelo funcional y mecanicista) que hay que descubrir para controlar (interés pragmático) esta nueva concepción.

Bajo la premisa anterior lo que se pretende investigar es una relación sujeto-objeto basado en el paradigma positivista, ya que busca la causa-efecto una explicación completamente lineal, la metodología que utiliza es cuantitativa y tiende a explicar el porqué de los hechos 
y no el para qué, la tradición Galileana nos describe que solo es posible conocer la realidad tal como se presenta (Galván, 2016).

La investigación cuantitativa normalmente está basada en alguna forma de positivismo lógico, el cual asume que hay hechos sociales estables, con una realidad única, separados de los sentimientos y de las opiniones de los individuos. Busca establecer relaciones y explicar las causas como objetivo de la investigación y el método en los estudios cuantitativos se recurre a un conjunto de procedimientos y pasos establecidos que guían al investigador, los estudios que lo caracterizan o prototipo es emplear diseños experimentales o correlacionarlos para reducir el error, el sesgo y las posibles variables extrañas y no olvidar que el papel del investigador ideal en este rubro permanece al margen del estudio para evitar los sesgos (McMillan \& Schumacher, 1996).

La garantía del conocimiento se basa en los mecanismos de la razón humana por lo que se precisan dos tipos de lógica en uso para la investigación educativa la lógica formal y la lógica dialéctica. En la concepción de la lógica formal lo que decide la validez de un razonamiento es el uso de un lenguaje estructurado, secuencial y matemático, la lógica probabilística y los enunciados condicionales son elementos clave dentro de este metalenguaje (Vélez, 2014).

La presente investigación se conceptúa en la lógica formal, propone explicar los determinantes de los procesos de acreditación de la escuelas y facultades de Medicina en México, por lo que se medirá y cuantificará su eficiencia para que logren el impacto que fundamentó su origen. La metodología cuantitativa se fundamenta en la construcción y medición de dimensiones, indicadores e índices de variables, y los datos deben responder a estos fenómenos, por lo cual tendrán validez si son verificables o no, lo cual quiere decir que deben ser observados y constatados de alguna forma. Es un proceso estructurado mediante un patrón preciso y predecible que relaciona y conecta sistemáticamente unas etapas con otras, las cuales no pueden estar aisladas, esto permite el tratamiento y confrontación de sus enunciados. El enfoque cuantitativo se orienta fundamentalmente a la medición de variables y sus relaciones, por lo cual deben ser verificadas para establecer la prueba de hipótesis y validar de esta forma las teorías anunciadas (Tamayo, 2017).

\begin{tabular}{|c|c|}
\hline \multicolumn{2}{|c|}{ TRADICIÓN GALILEANA } \\
\hline Paradigma & Positivista \\
\hline Método & Deductiva \\
\hline Enfoque & Empírico analítico \\
\hline Lógica & Formal \\
\hline Carácter & Cuantitativo \\
\hline
\end{tabular}

\section{Paradigmas de investigación}

Para Khun (1962), un paradigma describe (para una etapa específica en la historia de la ciencia) la manera cómo una comunidad científica como un área de estudio, identifica problemas apropiados como objetos de estudio y determina conceptos legitimados y métodos utilizados, esto es, la ciencia normal; el sentido de la realidad es temporalidad. El paradig- 
ma es un sistema de creencias, principios, valores y premisas que determina la comunidad científica de la realidad. Todo paradigma de investigación se basa en sistemas filosóficos y se operacionaliza mediante un sistema de investigación. Al ser una visión compartida por un grupo de científicos implica una metodología determinada, y debe precisarse para evitar el reduccionismo de legos en cuanto a su conceptualización.

Como se había comentado, el positivismo tiene antecedentes en los empíricos ingleses de los siglos XIII y XIX, cuya idea fundamental es el progreso del conocimiento de la sociedad con base en la experiencia observable. Además, la filosofía positivista de Augusto Comte afirma que se puede estudiar a la sociedad en forma racional y objetiva. Los enunciados sometidos a la lógica y a la verificación empírica transitan de neopositivismo al positivismo lógico del siglo XX, desde el cual se obtiene el tipo de investigación cuantitativa que integra a la estadística y sus ramas, como superación de la pseudociencia, enfatizando que el lenguaje científico es lenguaje matemático. Entendiéndose por científico solo aquello que es susceptible de ser medido y comprobado (Veléz, 2014).

En un breve análisis comparativo, para el paradigma positivista los intereses se centran en la predicción, el control y la explicación, mientras que para el paradigma dialéctico los intereses se enfocan en identificar el potencial para el cambio. En cuanto a la relación sujeto-objeto, en el primero existe una independencia total, pero, para el segundo, la interrelación es fundamental.

\section{Aspectos metodológicos de la investigación empírico-analítica}

La gnoseología, para las investigaciones empírico-analíticas, implementa el modelo de conocimiento mecanicista en la relación sujeto-objeto. Además, requiere revisar al empirismo clásico (F. Bacon, Locke, Hume), el racionalismo (Galileo y Descartes), el racionalismo crítico (Popper) y el empirismo lógico del neopositivismo del Círculo de Viena. Este enfoque de investigación está marcado por un estilo de pensamiento sensorial (empirismo), una orientación concreta y objetiva hacia las cosas, con lenguaje impersonal y matemático para conformar una vía analítica del conocimiento (racionalismo), hasta lograr la validación por medio de la realidad objetiva (realismo).

\section{DESARROLLO}

La discusión sobre el significado y la importancia de la calidad, ha llevado a reconocer en la evaluación y en la acreditación los medios que puedan contribuir a una reforma de la educación superior, de manera que, además, se constituyan en los canales adecuados para comunicar a los usuarios de las instituciones, sobre los niveles alcanzados en las funciones básicas (docencia, investigación y difusión cultural) que desarrolla una institución universitaria.

El establecimiento de un sistema de acreditación conduce a realizar un ejercicio de los fines, atributos y niveles de calidad que debe tener el sistema educativo en el mundo contemporáneo. Se requiere perfilar de manera colegiada, y con amplia participación social, el ideal educativo, con base en el análisis histórico, social, económico, político y cultural que, 
al mismo tiempo, concuerden con el proyecto del país y con un mundo cada vez más abierto e interdependiente, en el que se combinan cooperación y competencia.

De ese modo, acreditación, evaluación y calidad están estrechamente interconectados y resulta complejo considerar ambas variables por separado. Se acredita conforme a un proceso de evaluación y de seguimiento, con el fin de disponer de información fidedigna y objetiva sobre la calidad relativa de instituciones y programas universitarios, sea que estén en su fase de reconocimiento inicial o en pleno desarrollo de su proyecto institucional.

Una de las principales herramientas para garantizar que un programa académico de nivel superior es de calidad, es la acreditación porque implica que dicho programa ha sido evaluado, que aseguran su pertinencia y que este cumple con criterios y estándares de calidad previamente establecidos (Pallán, 1995). A finales del 2000 se constituye el Consejo para la Acreditación de la Educación Superior (Copaes). Se creó como una asociación integrada por personas morales y como una instancia capacitada y reconocida por la Secretaría de Educación Pública para conferir reconocimiento formal a favor de organizaciones cuyo fin sea acreditar programas académicos de educación superior que ofrezcan instituciones públicas y particulares, previa valoración de su capacidad organizativa, técnica y operativa, de sus marcos de evaluación para la acreditación de programas académicos.

El Copaes regula a los organismos que acreditan programas educativos; por lo que no acredita directamente programas, sino que da su aval a aquellas agencias de acreditación y para su desempeño se auxilia de un marco general para los procesos de acreditación de programas académicos de nivel superior, donde queda claro que un proceso de acreditación debe ser voluntario, integral, objetivo, justo y transparente; externo, ético y responsable; temporal, confiable, y producto de un trabajo de personas de reconocida competencia en la materia, lo que hace un proceso nada ajeno a lo que a nivel internacional se realiza, cada organismo acreditador posee sus propios criterios metodológicos para la acreditación, un marco de referencia e indicadores como costos del servicio, no obstante deberán ajustarse a lo normado por el Copaes (Morales, 2009).

Tabla 1.

Estado actual de la acreditación en México (Comaem)

\begin{tabular}{lccc} 
& PÚBLICAS & PARTICULARES & TOTAL \\
\hline Acreditadas & 43 & 46 & 89 \\
Acreditaciones vencidas & 5 & 5 & 10 \\
No acreditadas & 1 & 2 & 3 \\
Opinión favorable & 0 & 1 & 1 \\
Opinión no favorable & 1 & 1 & 2 \\
Sin procesos de acreditación & 10 & 34 & 44 \\
\hline \multicolumn{1}{c}{ TOTAL } & $\mathbf{6 7 0}$ & $\mathbf{8 9}$ & $\mathbf{1 4 9}$
\end{tabular}

Fuente: Comaem, https://www.comaem.org.mx/wp-content/uploads/2020/06/estadoglobal.htm 


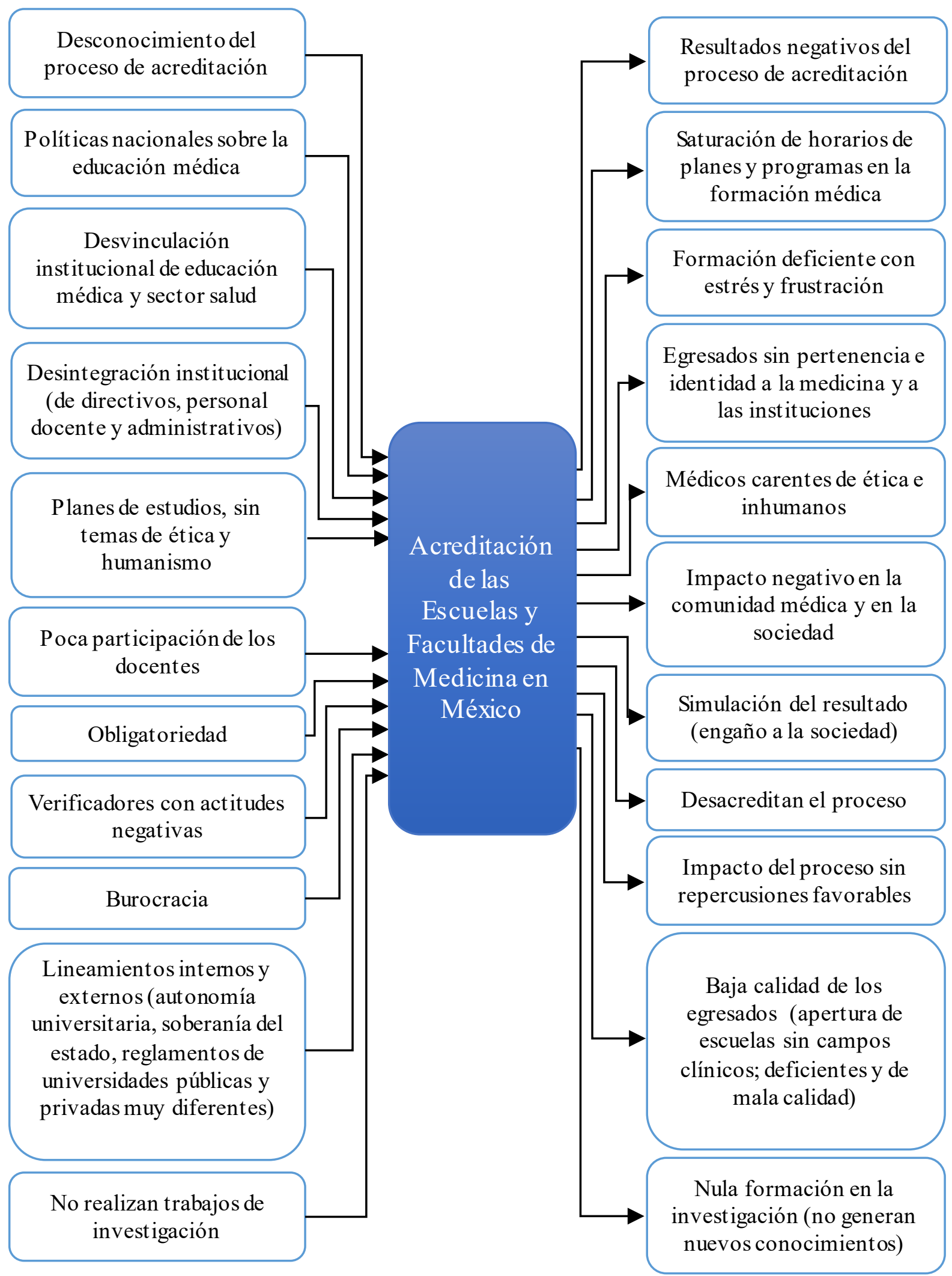


Figura 1. Análisis causa-efecto. Diagrama de Demócrito (Quien sustentaba que existen interconexiones de la realidad como cadenas)

En la actualidad la educación es un tema prioritario no solo para México, sino para un gran número de países. Todos se enfrentan a un grave problema cuyo origen es fundamentalmente económico o sociocultural; enfrentarse a los avances vertiginosos de la ciencia y la tecnología no ha sido tarea fácil y la medicina no está exenta de esta problemática. Actualmente contamos con servicios de salud públicos saturados y con un costo muy elevado del ejercicio de la medicina privada.

Resulta relevante conocer y analizar las circunstancias actuales del entorno nacional e internacional, la apertura del mercado laboral de servicios profesionales, así como las nuevas condiciones internas de las universidades que plantea la necesidad de flexibilizar, expandir, mejorar y hacer compatible el desarrollo de las instituciones del sistema de educación superior. En el marco de tales necesidades, los organismos acreditadores y los procesos de acreditación deben atender los requerimientos de las necesidades del sistema.

Las políticas actuales no suman a la calidad en la formación de los médicos, sin embargo los educandos abonan, sobremanera, a la salud de la población mexicana. Existen hospitales que gracias a los estudiantes de internado y principalmente a los médicos que cursan las diferentes especialidades como residentes médicos; cumplan con su misión. Sin embargo, el exceso de trabajo, la saturación de tareas y las revisiones bibliográficas dan origen a la fatiga constante, lo que repercute negativamente en los resultados esperados en su formación.

Con motivo de los cien años de la Constitución de México se llevó a cabo un simposio sobre la constitución y la salud. El ponente Sánchez Mejía, de la Suprema Corte de Justicia de la Nación, manifestó:

Me gustaría comenzar comentando algo que ustedes viven día a día que ya lo saben la salud se ve impactada necesariamente por muchos factores, el avance científico, el avance tecnológico e incluso efectos biológicos o ambientales, lo cual implica que el derecho a la salud sea un derecho dinámico, y este dinamismo en el derecho a la salud implica o genera dos cosas: por un lado, un efecto normativo, y por otro, un efecto interpretativo.

Cuando un programa de estudios se somete a este sistema de evaluación, la Asociación Mexicana de Facultades y Escuelas de Medicina (Amfem), al final, emite un dictamen oficial que puede ser: acreditado, no acreditado o acreditación condicionada al cumplimiento de recomendaciones académicas específicas. Este resultado no es confidencial. Por tanto, es publicable y de interés en la medida en que muchas personas desearán saber, por ejemplo, si la escuela o facultad donde han decidido realizar sus estudios de medicina está o no acreditada por la Amfem. De esta manera, se convierte en un indicador de calidad relevante para el desarrollo del país y para las aspiraciones personales del profesional en formación.

Liftshitz \& Zerón (2010), en su estudio, determinan que la educación socialmente se reconoce como el camino para alcanzar el progreso individual, grupal y comunitario; en el caso de la atención a la salud, la formación docente y la educación continua del personal son 
fuente rica para contribuir a elevar la calidad de los servicios médicos y agrega que la educación médica no es un sistema cerrado; por tanto, su autonomía es limitada y relativa. La determinación de su cosmovisión, estructura formal, infraestructura y prácticas docentes ha estado y está potentemente influida por la ideología -valores, visiones, expectativas-y por las prácticas dominantes en el sistema de atención médica. Entender esto es un preámbulo fundamental para comprender la modernización y la innovación de la educación.

Después de la Segunda Guerra Mundial, la Organización Mundial de la Salud acuñó: "La salud es un estado de completo bienestar físico, mental y social, y no solamente la ausencia de afecciones o enfermedades". ${ }^{2}$ Consecuente con ello, la Organización Panamericana de la Salud (OPS) organizó dos seminarios, uno en Viña del Mar, Chile, y otro en Tehuacán, México; de los que surgió la exhortativa a las escuelas de medicina del continente para que incluyeran en sus planes de estudio la enseñanza de la medicina preventiva y social. Esta recomendación fue acogida por numerosas instituciones de enseñanza médica; sin embargo, enfrentó resistencia activa de los que se opusieron abierta o pasivamente y de los que simplemente la ignoraron, los estudiantes, en general, consideraron que esta educación era un requisito molesto que había que cumplir.

De acuerdo con las recomendaciones de la OPS, se incluyeron asignaturas de medicina preventiva, sociología y psicología médica. Sin embargo, el paradigma expectativo dominante estaba en contradicción lógica con esta enseñanza, puesto que realizar la prevención de la enfermedad requiere, sine qua non, la anticipación del evento enfermedad. Así, la enseñanza en el ambiente hospitalario se vio condicionada a la imposibilidad lógica de actuar con anticipación y, por ende, a realizar la prevención de la enfermedad y la protección de la salud.

Liftshitz \& Zerón (2011) manifiestan que el foco de atención de la capacitación son las normas y los procedimientos, entre otros. Los profesores naturales son los directivos, que son quienes los emiten y deben vigilar su cumplimiento. Por su parte, la actualización debiera ser más selectiva y encauzarse al personal que culminó previamente a un proceso de formación y tiene un perfil profesional. Son ejemplos: médicos, enfermeras, técnicos, por decir algunos. El proceso educativo se dirige frecuentemente para que los educandos acumulen información sin debatir su valor o pertinencia.

Malpica (2012) se pregunta ¿Es posible determinar si una práctica educativa es más o es menos adecuada? En este siglo XXI, todo parece indicar que el prestigio social de profesionales de la educación y la formación médica se irá poniendo cada vez más en duda. Sin embargo, la sociedad no puede prescindir del personal médico y el rol del profesional, así como su participación en el presente y futuro de las sociedades, dependerá de las respuestas del profesional y de las capacidades profesionales que se formen durante la práctica educativa.

Ruelas \& Concheiro (2010), en su libro, hace un intento de reflexión ordenada y amplia sobre la posible evolución de la salud en México en los próximos 40 años. Propone una exploración de algunos de los futuros posibles tanto de los condicionantes de la salud como el propio sistema de salud. Incluye también algunas reflexiones sobre lo que podría ser 
deseable que ocurriese al interior del Sistema Nacional de Salud. El libro no pretende plantear lo que ocurrirá de aquí al año 2050, sino especular sobre lo que podría ocurrir o sería deseable que ocurriese, con el fin de establecer posibles puntos de referencia que permitan tomar decisiones desde ahora para orientar al sistema hacia el futuro.

Tena \& Hernández (2005) hacen énfasis en la comunicación humana específicamente en la relación médico-paciente y mencionan que al médico "le damos" el nivel de educando permanente, debido a que, por la naturaleza intrínseca de su profesión, más que de cualquier otra, requiere del estudio en forma permanente, para mantenerse alineado a los adelantos científicos, tecnológicos o humanistas, para el ejercicio práctico de su profesión, así como para ejercer la docencia. Por lo anterior se establece un símil entre el médico y el docente, el aula y el consultorio, la forma de comunicación o relación personal en el aula, como el ambiente creado en ella como dentro del consultorio, y la responsabilidad que se piensa debe tener un buen maestro para con los resultados del aprendizaje, logrado con sus alumnos o pacientes.

\section{CONCLUSIONES}

El sistema para la acreditación de la educación médica en México está sujeto a interconexiones con la realidad donde cada acción conlleva a un resultado predecible. Por lo tanto, si existe un desconocimiento o un desentendimiento del proceso de acreditación por parte de las facultades de medicina del país; tendría un impacto negativo en la acreditación de los programas de estudio. Igualmente, de darse una desvinculación institucional entre la educación médica y el sector salud, traería como consecuencia una formación médica fragmentada y frustrante, carente de las capacidades necesarias para enfrentar la complejidad del problema de la salud y la educación.

Al mismo tiempo, resulta de vital importancia que los planes de estudio diseñados para la formación de los profesionales de la salud, cuenten con la ética y el humanismo como temas transversales porque de lo contrario podría contribuir con la formación de profesionales carentes de ética y de solidaridad hacia el paciente incapaces de demostrar empatía y compromiso con el paciente.

Finalmente, otro elemento decisivo es la investigación científica. Si durante la formación médica, la investigación no se sitúa dentro de las funciones esenciales del docente y del alumnado, automáticamente se estaría limitando la capacidad del descubrimiento y por ende la generación de conocimientos nuevos útiles aplicables al ejercicio profesional.

\section{REFERENCIAS}

Galván, N. (2016). Trabajo de teoría del conocimiento y lógica. Universidad de Cartagena.

Khun, T. (1962). La estructura de las revoluciones cientificas. 1982. FCE.

Liftshitz, A., \& Zerón, L. (2010). Los Retos de la educación médica en México. Academia Nacional de educación Médica y DPC de México A.C. 
Liftshitz, A., \& Zerón, L. (2011). Los Retos de la educación médica en México. Academia Nacional de educación Médica y DPC de México A.C.

Malpica, F. (2012). 8 Ideas clave. Calidad de la práctica educativa. Ed. Grao.

McMillan, J., \& Schumacher, S. (1996). Investigación Educativa (5ª edición). Pearson Addison Wesley

Morales, R. (2009). La acreditación internacional de programas educativos, como estrategia para lograr la calidad en la Educación Superior. Universidad Autónoma de México.

Pallán, F. C. (1994). Los procesos de evaluación y acreditación de las instituciones de educación superior en México en los últimos años. Revista de la Educación Superior, 23(3), 1-20. http://publicaciones.anuies.mx/pdfs/revista/Revista91 S1A1ES.pdf

Ruelas, E., \& Concheiro, A. (2010). Los futuros de salud en México 2050. El Atril Tipográfico.

Tamayo, M. (2017). El proceso de la investigación cientifica. (5ta edición). LIMUSA.

Tena, C., \& Hernández, F. (2005). La comunicación humana en la relación MédicoPaciente. Prado.

Vélez, D. (2014). Perspectiva epistemológica para la investigación educativa: aspectos fundamentales, técnicos y metodológicos. Grupo Ed. EXODO. 